\title{
Energy generation based on piezoelectric transducers
}

\author{
J. Ortiz ${ }^{1}$, N. Zabala ${ }^{1}$, P. M. Monje ${ }^{1}$, V. Cokonaj ${ }^{2}$ and G. Aranguren ${ }^{1}$ \\ ${ }^{1}$ Electronic Design Group \\ University of the Basque Country \\ Faculty of Engineering of Bilbao - 48013 Bilbao (Spain) \\ Phone: +00 3494 6017369, e-mail: javier.ortiza@ehu.es \\ ${ }^{2}$ AERnnova Engineering Solutions Ibérica S.A \\ Av. Manoteras, 20 B-5 ${ }^{\mathrm{a}}$, 28050 Madrid (Spain)
}

\begin{abstract}
In this paper, the design, implementation and testing of a prototype for electric energy generation from collecting wasted ambient power sources coming from the mechanic vibrations (Piezoelectric Energy Harvesting $\mathrm{PEH})$ is presented. This prototype has been specifically designed for applications where it is required to connect multiple transducers in order to obtain greater power levels. This design is based on a previous comparative analysis among all the existing auto-harvesting circuits. The prototype has been tested both in laboratory tests as in real field trials on board railway units, with encouraging results. It has been proved that, though achieving power levels of a few milliwatts, its application in the autonomous low-consumption devices powering systems field is a real possibility.
\end{abstract}

\section{Key words}

Energy Harvesting, Piezoelectric Energy Harvester, Power Management Circuits, Resonant tuning.

\section{Introduction}

The energy harvesting is the process of collecting not-used environmental energy and makes the most of it by generating electric energy which can provide a very small amount of power for low-energy electronics. We could name this kind of process as "the little sister of the renewable energy".

Energy harvesting technologies is attracting an increasing interest among the research community due to its patent possibility for substituting low-consumption electronic devices powered by batteries. For instance, energy harvesting could be used in nodes of wireless network or in microelectromechanical sensors (MEMS), which require extremely low power levels, around a few microwatts.

Electronic devices mobility is another key factor that determines the need for renewable energy sources, so that they can be transported easily with the electronic system while performing their energy collection task.
The existing researches among the scientific community have focusing on building generating prototypes at different scales, which varies from the nanometric to the macrometric scale, always with the purpose of powering these electronic devices. In addition, nowadays these researches are centered on increasing the application field range of these technologies to a greater number of devices, which total consumption requirements are around the level of a few milliwatts. With the objective of designing a system that works in this range, the project presented in this paper presents an autonomous energy harvesting electronic system able to recollect energy using multiples piezoelectric transducers.

Section 2 provides a general overview about energy harvesting technologies and, especially, about piezoelectric based vibrational energy harvesting. General schematic of an energy harvesting system is discussed in Section 3, paying special attention to the Energy Management block and the different alternatives available. Experimental setups are presented in Section 4, experiments carried out with both one and more than one piezoelectric transducers. In concluding section summary and a perspective on further research is presented.

\section{Energy Harvesting Overview}

Energy harvesting, or energy scavenging, is defined as extracting energy from ambient environment. The ambient energy to be transformed into electrical energy can be in form of light, thermal gradients, kinetic or radiofrequency $(\mathrm{RF})$ energy.

Solar power provides the most familiar form of energy harvesting. Based on the photovoltaic effect this technology is able to produce up to $10 \mathrm{~mW} / \mathrm{cm} 2$ on a sunny day. Solar photovoltaic panels, however, are not suitable for locations where light is not available or it is not possible to position a panel orientated to the sun.

Mechanical vibrations are present in a number of useful locations with the advantage of no restriction about size and need of light. There are three main transduction mechanisms for the task of converting mechanical energy 
into electrical energy: electromagnetic (EM), electrostatic (ES) and piezoelectric (PZ).

1) Electromagnetic: Based on Faraday's induction law, electromagnetic coils produce higher powers, available ranges are from $20 \mu \mathrm{W}$ to $200 \mathrm{~mW}$ [1], but are generally larger in size and the generated output voltages are low $(<1 \mathrm{~V})$ requiring further conversion stages.

2) Electrostatic: Electrostatic devices using a type of variable capacitor to harvest the energy [1] produce power levels of (10 to $40 \mu \mathrm{W})$ much lower than electromagnetic devices and to be operative require an external electrical source such as a battery.

3) Piezoelectric: Piezoelectric transducers, are well suited for miniaturization, generate suitable output voltages up to $20 \mathrm{~V}$, but with very high output impedance, which means levels of output currents in the range of microamperes providing levels of power up to a few tens of milliwatts.

A summary of power levels achieved for vibrational energy harvesting sorted by type of transduction is shown in Table I.

Table I - Power levels for vibrational energy harvesters [2].

\begin{tabular}{|ccccc|}
\hline Type & $\mathrm{P}(\mu \mathrm{W})$ & $\mathrm{F}(\mathrm{Hz})$ & $\mathrm{A}\left(\mathrm{m} / \mathrm{s}^{2}\right)$ & $\mathrm{P}\left(\mu \mathrm{W} / \mathrm{mm}^{3}\right)$ \\
\hline EM & 2000 & 100 & 1.0 & 0.04 \\
EM & 3000 & 60 & 1.0 & 0.05 \\
ES & 24 & 30 & 10 & 0.015 \\
ES & 8 & 50 & 8.8 & 0.21 \\
PZ & 375 & 120 & 2.5 & 0.18 \\
PZ & 3060 & 50 & 15.3 & 18 \\
PZ & 160 & 80 & 2.3 & 0.25 \\
\hline
\end{tabular}

According to their features, piezoelectric vibrational harvesting is probably the most promising solution. However, this is a technology with some constraints which limit its efficiency. First, as most of the resonant systems, has a narrow frequency response which means that only when external vibration frequency is close to his resonant frequency produces power. In other words, in order to optimize energy harvesting the natural frequency of the piezoelectric needs to be matched to the vibration frequency of the structure that the piezoelectric is mounted to. Secondly, the high internal impedances require adapted energy management electronic hardware to reach optimum transmission of power. Lastly, the alternating nature of the generated currents must be rectified to be unidirectional as it's demanded by electronic devices.

Typical materials employed in energy harvesting are synthetic ceramics such as Lead Zirconate Titanate (PZT) or, recently developed, Macrofiber Composites (MFC), both of them manufactured in thin sheet-form because of its mechanical advantages and its low cost. However, ceramic materials are fragile and it is possible that, in cases of being submitted to high-amplitude vibrations during a long time, they can break themselves. In order to avoid these damages, a recent solution has been to insert more resistant material's sheet between the PZT sheets, in order to reinforce them.

\section{Energy Harvesting Designed System}

A typical energy harvester system consists of several subsystems, which includes Energy Transducers, Energy Management hardware and Energy Storage to provide energy to the electronic application under suitable and stable voltage specifications. A typical energy harvester system is shown in Fig. 1.

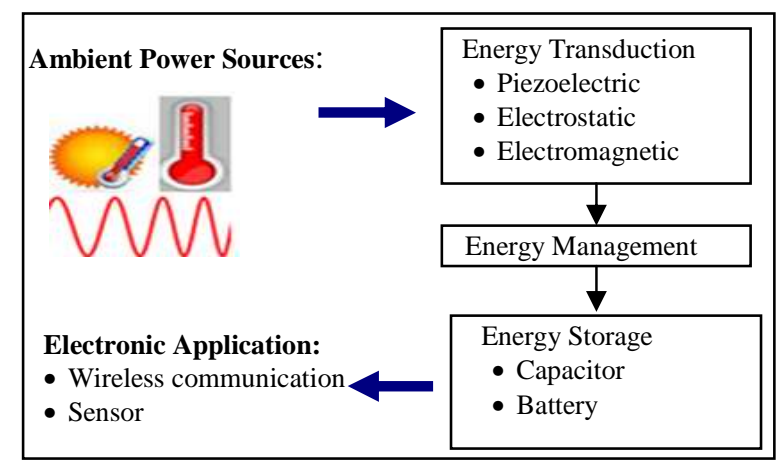

Fig. 1. Generic Energy Harvester System Schematic.

The simplest circuit for Energy Management is the diodebridge rectifier but its efficiency is very dependent of the electrical load. Attempts have been made to design circuits which improve diode-bridge performance. There are two solutions for this problem. The first option is being carried out by Guyomar et al. [3], who have developed a technique called Synchronized Switch Harvesting on Inductor (SSHI), which is based on commuting the piezoelectric over an inductor in the moment of maximum electric energy harvested in the piezoelectric. This SSHI technique can be implemented with many configurations, which are called parallel SSHI, series SSHI and Synchronous Electric Charge Extraction (SECE). Recently some other groups [4-5] have proposed innovative schemes leading to better performance. Each of these configurations is defined by the relative position of the pair switch-inductor. The implemented techniques include self-switching techniques based on transistors and diodes which avoid the need of external control circuits or energy sources. However, inductors and self-switching circuits entail an excessive size. Therefore, the use of these techniques is limited to one piezoelectric.

The other solution proposed by different authors [6-8] presents the insertion of a DC/DC converter between the diode rectifier and the capacitor. So that the rectifier works in its optimal working mode with a voltage in its rectified output $V_{\text {Rect_opt }}$ equal half the open-circuit voltage $\mathrm{V}_{\mathrm{OCp}}$ in the piezoelectric. To that effect, the DC/DC converter must not only regulate the output voltage but offer optimal input impedance to the rectifier. However, in most cases the vibrations' amplitudes and, therefore, $\mathrm{V}_{\mathrm{OCp}}$ voltages generated by the piezoelectric are variable. 
Trying to combine both objectives implies to design overly complex circuits and with inacceptable consumption requirements. Nevertheless, for certain applications where vibrations' amplitudes are previously known and practically constant, it could be interesting to design a convertor that fixes the output voltage of the rectifier in the optimal value.

An intermediate solution consist of using a diode bridge followed by a commercial DC/DC converter with a minimum working voltage close to the optimum output voltage $\mathrm{V}_{\text {Rect_opt }}$ in the rectifier. With this idea in mind, manufacturer Linear Technologies has developed and marketed a circuit designed for auto-harvesting applications with high-impedance transducers: LTC35881. This integrated circuit have a low-loss, it is a full-wave bridge rectifier with a high efficiency buck converter to form a complete energy harvesting optimized for high output impedance energy sources, such as piezoelectric transducers. In addition this integrated circuit features a regulated maximum output current which results very useful when working various integrated circuits in parallel.

In order to verify the performance of these circuits, they were subjected to a comparative analysis. For that, four circuits were selected and tested. Those are: diode bridge (used as the reference in this analysis), series SSHI rectifier combined with Texas Instruments TPS62120 (High efficient DC/DC converter), and integrated circuit LTC3588-1. The test consisted of applying to each of four circuits the same electrical signal, which emulates the one supplied by a piezoelectric transducer.

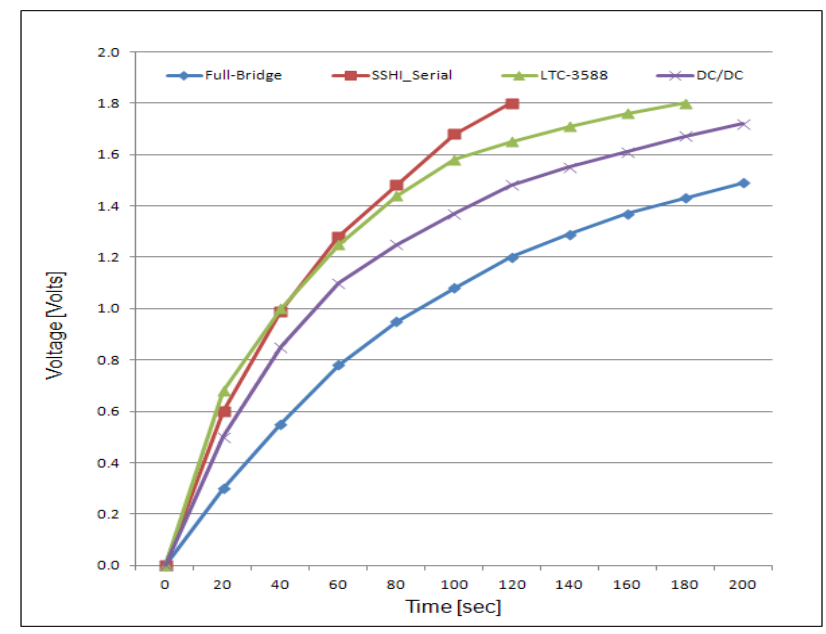

Fig. 2. Voltage versus time curves for the four types of circuits.

This emulation of the piezoelectric transducer was accomplished by its Thevenin equivalent which was previously validated after comparative tests with a piezoelectric. For that, the piezoelectric transducer was subjected to vibrations of $20 \mathrm{~Hz}$ and the output voltages were measured after connecting different resistive charges. This process was repeated changing the amplitudes of the vibrations applied. As can be seen in Fig. 3 (b), the differences between the obtained voltages using the Thevenin equivalent and those generated by the piezoelectric makes it possible to approach this research using the Thevenin equivalent in a versatile way with enough approximation to the real process.

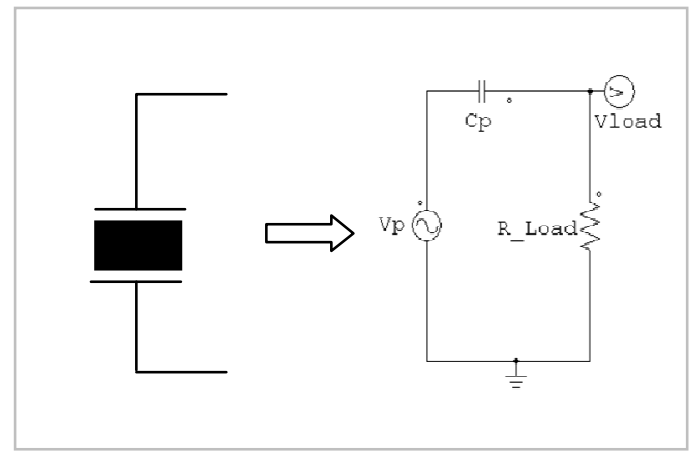

(a)

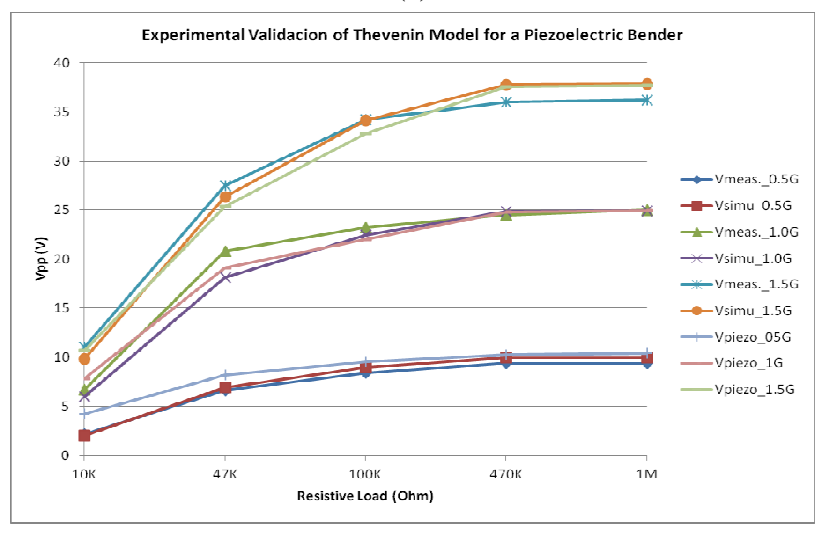

(b)

Fig. 3. (a) Piezoelectric symbol and its Thevenin equivalent.

(b) Test signals for validation of the Thevenin equivalent.

Results of this test with the four circuits are shown in Fig. 2. SSHI series are the best option with one channel while LTC3588-1 leads to combination of rectifier and DC/DC converter. After new rounds of test, it was checked that, with input voltage levels greater than $20 \mathrm{~V}_{\text {peak-to-peak, }}$, this comparative is even more favourable to LTC3588-1, although when those inputs were lower than $10 \mathrm{~V}$ peakto-peak the performance decreased slightly.

In a second round of tests the objective was to measure the feasibility and efficiency of each of these circuits to be grouped to work in parallel. To this end, each circuit was connected with another identical circuit to form groups of two, three and four circuits working in parallel, simulating various piezoelectrics disposed at the same time. Once again each of the circuits received a $20 \mathrm{~V}$ peak-to-peak sinusoidal signal with $20 \mathrm{~Hz}$ frequency and in each circuit's output, a $10 \mathrm{mF}$ capacitor was charged from $0 \mathrm{~V}$ to $1,8 \mathrm{~V}$.

Results of this second round of tests are shown in Fig. 4 in terms of average power relative to one channel charging capacitor connected to full-bridge circuit. The integrated circuit LTC3588-1 is the best option for applications with multiple piezoelectric transducers because it is fully scalable. To each new channel added its performance increase exactly in a linear mode. Further tests using as much as 96 channels have verified this beneficial circuit behaviour. Furthermore, it was found that, in spite of working in parallel, the maximum charge 
current of the capacitor remains limited to a constant value low enough to favour the lifetime of the capacitor.

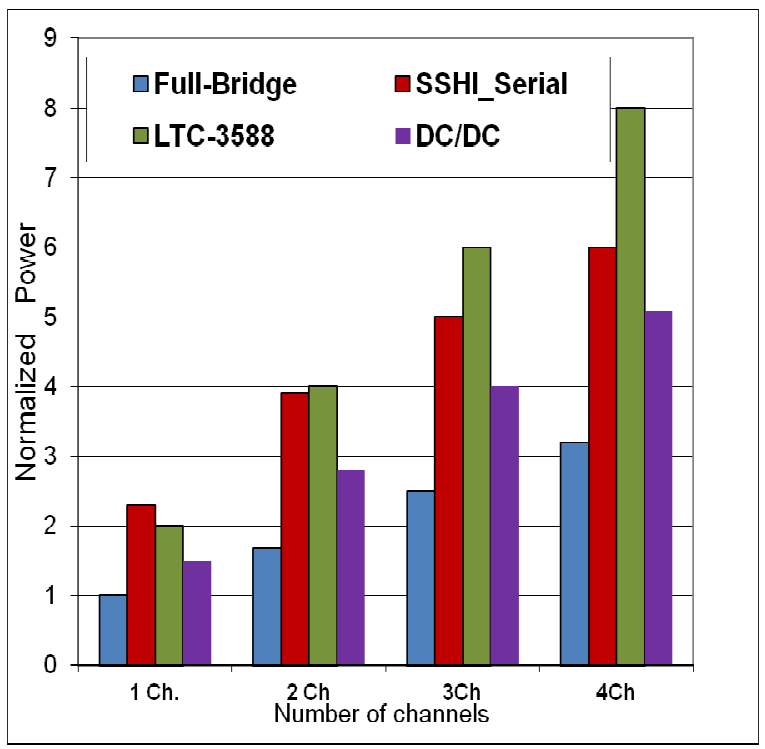

Fig. 4. Comparative of Average Power for different energy harvesting circuits using multiple channels.

Moreover, downsizing is another important factor that favours the performance of this circuit. The data in Table II below, showing size in $\mathrm{mm}^{3}$ of each energy harvesting cell, demonstrates this assertion.

Table II. - Sizes in $\mathrm{mm}^{3}$ of tested circuits.

\begin{tabular}{|c|c|c|c|c|}
\hline Circuit & FullBridge & SSHI_serial & LTC3588 & DC/DC \\
\hline Size $\left(\mathrm{mm}^{3}\right)$ & 80 & 350 & 120 & 180 \\
\hline
\end{tabular}

With these results in mind, a new integrated electronic device has been designed with capacity for 24 channels. These 24 channels have been organized into eight threechannel groups. In each group and at the same time, three circuits work in parallel charging a $10 \mathrm{mF} 1.8$ Volts maximum-voltage supercapacitor. All eight groups are connected among them in a serial configuration so that the output range goes from $1.8 \mathrm{~V}$ to $14.8 \mathrm{~V}$. In the diagram in Fig. 5, the block diagram of this integrated electronic system, called PEH-PM24 (Piezoelectric Energy Harvester Power Management 24 channels), is represented.

The PEH-PM24 electronic system can be configured in many modes in order to obtain more or less voltage or more or less power, in function of the number of piezoelectric transducers connected to the device or in function of the disposal of those transducers. For instance, in case of connecting one transducer (the minimum number), the power obtained is the lowest of all and the voltage is only of 1.8 Volts. When connecting three transducers at the same group or parallel connection, 1.8 Volts are obtained again but with a power three times greater than in the previous case. If eight piezoelectric transducers are connected each one to one group or serial connection, this electronic device can obtain up to 14.4 Volts with minimum power. In the case of using 24 piezoelectric transducers (one for each input), 14.4 Volts with a maximum power are obtained. Among all these configurations, many others are available.

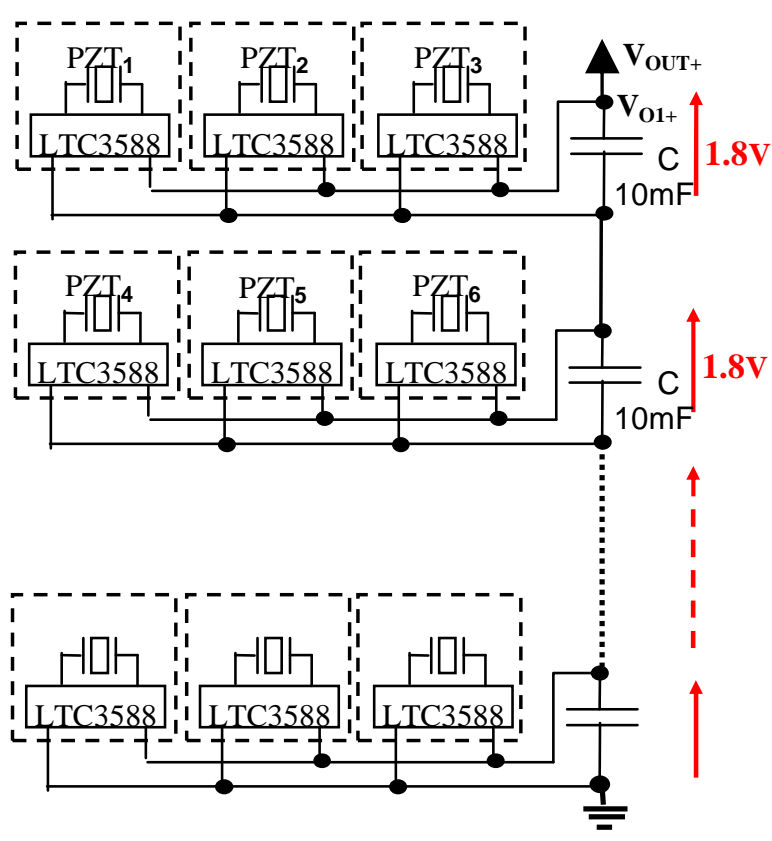

Fig. 5. Block diagram of the PEH-PM24 electronic system.

One advantage of using these transducers is that they can be connected among themselves both in serial and in parallel configuration. There are many references [9] of tests performed with multiple piezoelectric transducers in aeronautical applications where it is shown experimentally that in states with limited amplitude's vibration the serial connection is the best of all. Naturally, this is only possible when the transducers to be connected are subjected to vibrations practically equal and the generated voltages are tuned.

\section{Experimental Setup}

In order to test the working of the manufactured electronic device, real tests have been carried out using piezoelectric transducers. These tests have been performed in two separated stages. The first one will be employing only one transducer and the second will with multiple transducers.

The experimental tests have been performed using a shaker where the piezoelectric were placed in a cantilever configuration. A variable frequency and amplitude exciting signal were applied to the transducer. Piezoelectric transducers' electrodes were connected to every input channels of the PEH-PM24 and the voltage in the capacitor values were acquired in an oscilloscope, from 0 Volts to 1.8 Volts. The experimental setup is shown in Fig. 6 (a).

In this round of tests it was possible to compare and characterize both types of piezoelectric transducers commonly used: PZTs and MFCs. Average generated power curves for both types are shown in Fig. 6 (b). MFC transducers generate $10 \%$ more power than PZT. However, PZT transducers obtain a greatest power density (measured in $\mathrm{mW} / \mathrm{mm}^{2}$ ). 


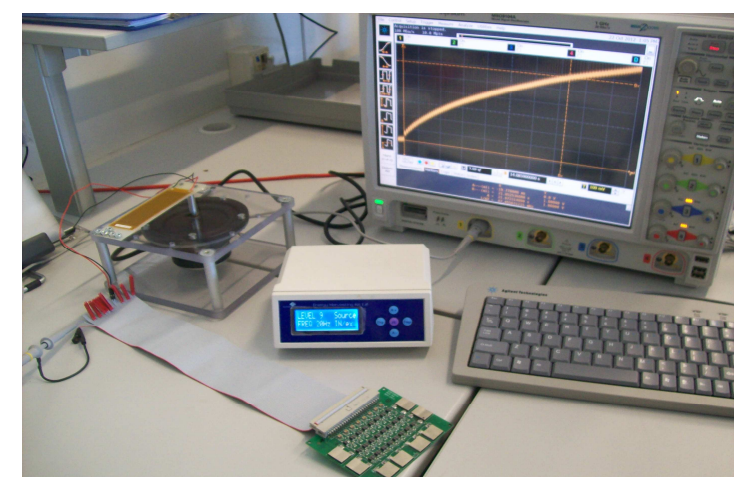

(a)

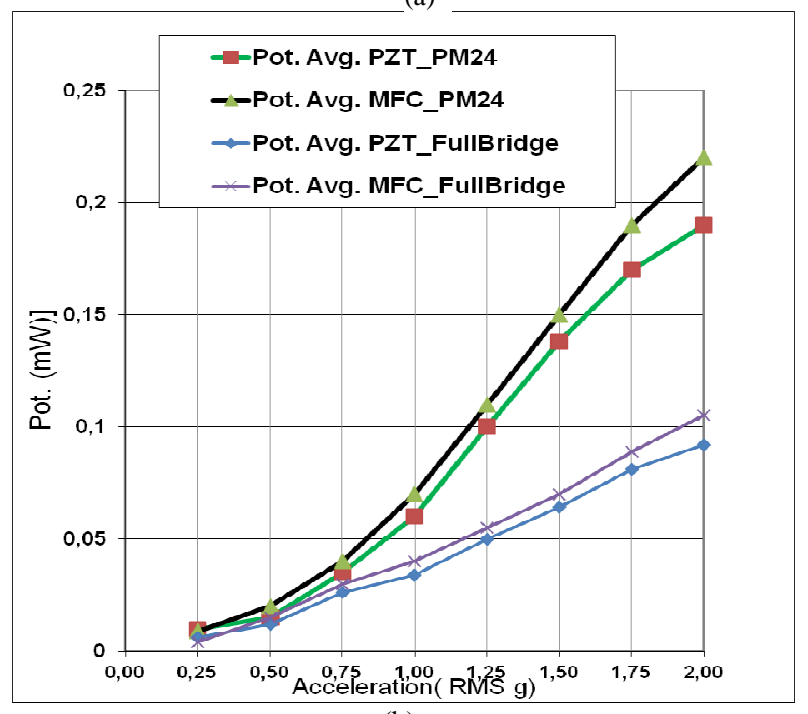

(b)

Fig. 6. (a) Components of the experimental setup with one piezoelectric (b) Average Power curves for both types: MFC and

PZT. Comparison between PEH-PM24 energy management circuit and diode full-Bridge.

Moreover, power obtained by both Energy Management circuits, PEH-PM24 circuit and full-bridge circuit, has been compared. These results have confirmed the results obtained in previous tests based on the piezoelectric's Thevenin equivalent.

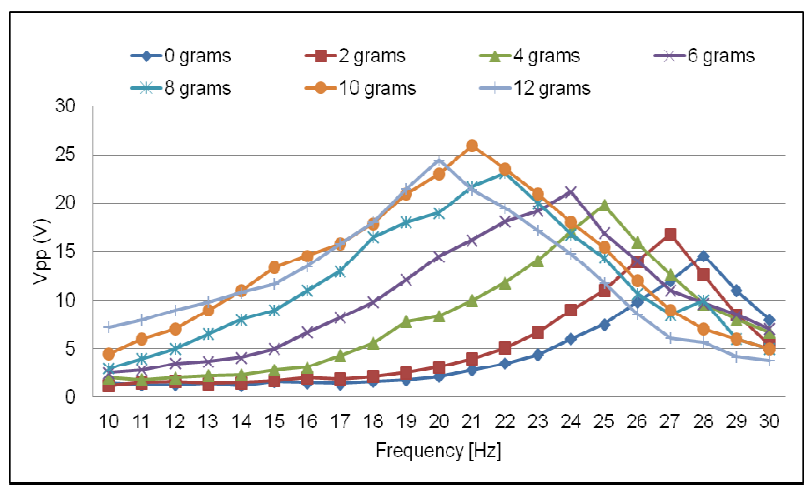

Fig. 7. Voltage-frequency response to the vibrational excitation in function of the weights added to the transducers.

The performance or ratio between generated electric energy and the input mechanic energy depends fundamentally on the correct tuning between two frequencies: external mechanical vibration frequency and piezoelectric natural vibration frequency. This last one can be modified by placing inertial masses in the open end of the piezoelectric transducer. The lower the weight in the piezoelectric, the lower natural frequency and, therefore, the generated voltage will be higher, as shown in Fig. 7. In that figure, the same piezoelectric transducer with different masses is subjected to harmonic vibrations under the same conditions of acceleration and different conditions of frequency. When excited by an acceleration of 1 grms, the obtained peak-to-peak voltage in opencircuit is 25.5 Volts, corresponding to a ten grams weight. As this mass' weight is reduced, the generated voltage is lower and the natural frequency obtained is higher.

Tests performed with multiple piezoelectric transducers have been carried out in a mechanizing center (Kondia B700 milling machine), ruled by numeric control equipped with a D'Andrea TA-125 (Fig. 8). This equipment will be able to generate the desired exciting frequencies and accelerations. To check the operation of the auto-harvesting design, a test prototype has been built, denominated Piezoelectric Energy Harvester Box (PEH_box), which includes the PEH-PM24 device connected to eight transducers (T215-A4-503X, de Piezo Systems manufacturer).Specifically, this model of piezoelectric is one with largest size but provides the highest power density. The selection of the monolithic piezoelectric instead of the flexible MFC type is, precisely, due to its highest power density in vibration applications.

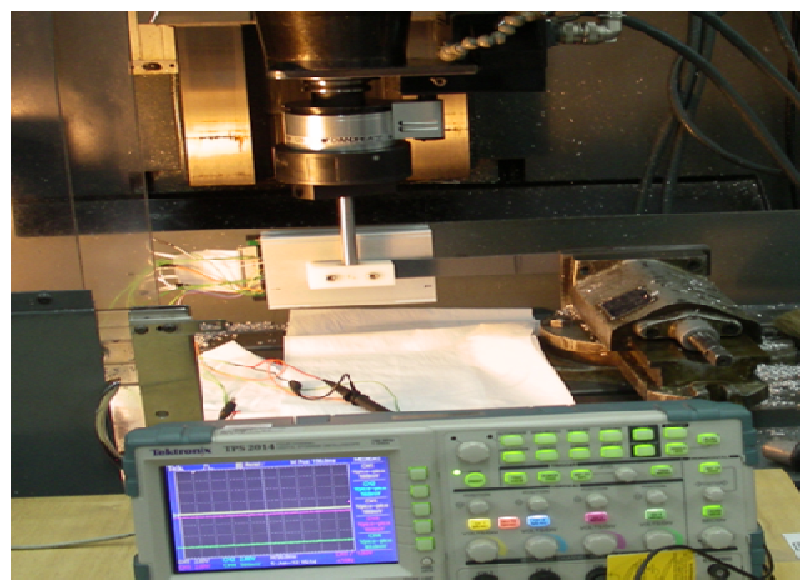

Fig. 8. Setup for multiple piezoelectric testing.

Piezoelectric transducers have been placed physically in two four-transducer parallel rows over a very rigid metallic frame, where only one end of the piezoelectric is fixed to the structure, leaving the other end free. That is known as a cantilever configuration. All these components are enclosed into an aluminium box and the fully assembled system is suitable for a direct installation, ready for experimental tests. This setup is shown in Fig. 9. Over the PEH_box, a high-performance tri-axis inertial MEMS digital accelerometer has been fixed. Using this accelerometer, it will be possible to measure the acceleration suffered by the box during these tests and, afterwards, compare these results with others present in the scientific literature.

In order to verify that the tuning was appropriate and correct, two different sets of piezoelectric transducers were used. In the first one, ten grams masses were placed 
in their free ends. The second ones did not carry any masses in them. These ten gram masses obtain a good tuning between the transducers' frequency and the 20 Hertz external frequency. A 0.7 grms acceleration and a 20 Hertz frequency were the characteristics of the excitation vibration.

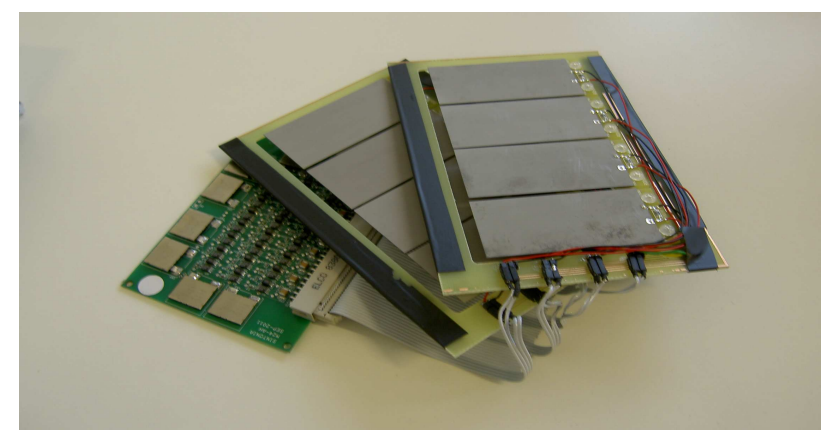

Fig. 9. PEH-PM24 card and groups of piezoelectric for prototype.

The generated capacitor`s voltage curves are shown in Fig. 10 , where the voltage in the capacitor each five-seconds is represented. As it is shown, the four $20 \mathrm{~Hz}$ tuned transducer group has a faster load than the free one.

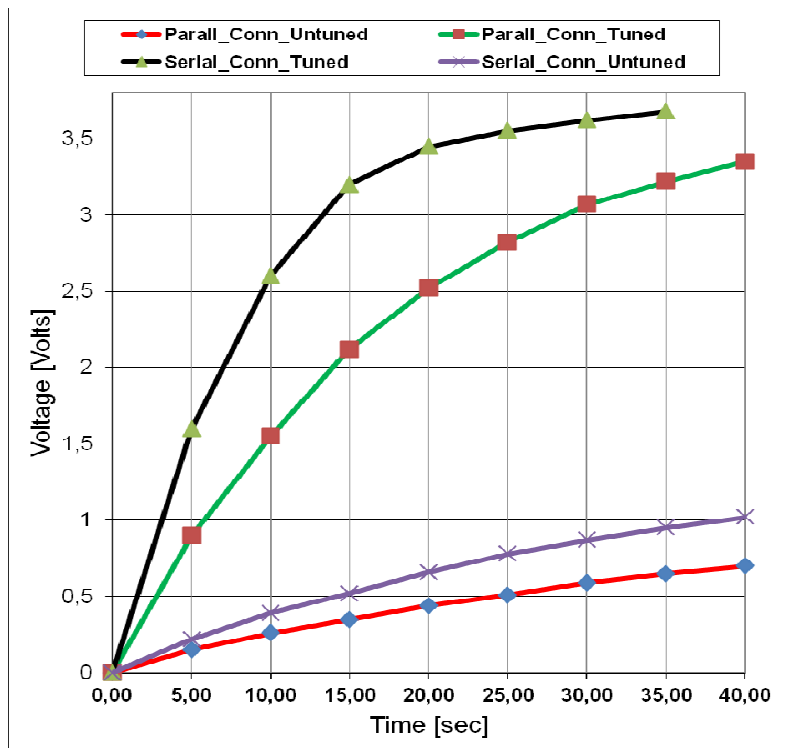

Fig. 10. Generated voltage curves obtained when using multiple piezoelectric transducers.

Moreover, another test was performed connecting, two by two, the piezoelectric transducers among themselves. The parallel connected and tuned piezoelectric transducers group increases the generated voltage from 1.5 Volts to 2 Volts in 4.1 seconds. However, when the piezoelectric are connected in serial mode, this same load happens in 2.3 seconds. Studying these curves and calculating the immediate powers, it is obtained that, for the serial connection, the maximum power is generated from 2.5 Volts to 3 Volts. The period of time needed for that is three seconds, which translates into an average power of 0.6 miliwatts. Taking into account the lineal scalability of the Energy Management electric device, in case of installing two devices instead of only one, in the same frame of time it would be possible to obtain a load of 5 or 6 Volts. Consequently, as capacitors are being charged with higher voltages and being the power proportional to the voltage to the square, the obtained power will rise up to $2.29 \mathrm{~mW}$.

\section{Conclusions}

It has been proved that piezoelectric transducers are a good option when generating low levels of power with the objective of powering up electronic devices, in vibration environments.

The greatest levels of power are obtained using multiple piezoelectric transducers tuned to the vibration frequency of the structure where they have been bonded to.

The experimental tests have been also extended to the monitoring of bogie variables in railway tracks. In future works, this research line will be deepen in order to obtain a practical application that can be used practically both in railway and in aeronautical environments.

\section{Acknowledgement}

The authors are grateful to Spanish Government for funding this project (CENIT SINTONIA), to The Basque Government for funding this research (ETORTEK AIRHEM-II) and to Metro Bilbao (www.metrobilbao.net, Technical Department) for allowing the use of their infrastructure and railway units during the on-board tests.

\section{References}

[1] C. B. Williams and R. B. Yates, "Analysis of a Microelectric Generator for Microsystems", in Sensors and Actuators 1996, Vol. 52, pp. 8-11.

[2] D. J. Inman and S. Priya, Energy Harvesting Technologies, Virginia Tech Center for Intelligent Material Systems and Structures (2009).

[3] D. Guyomar, Y. Jayet, L. Petit, E. Lefeuvre, T. Monnier, C. Richard and M.Lallart, "Synchronized switch harvesting applied to selfpowered smart systems: piezoactive microgenerators for autonomous wireless transmitters", in Sensors Actuators 2007, Vol. 138, pp. 151-160.

[4] S. Mehraeen, S. Jagannathan, and K. Corzine, "Energy harvesting from vibration with alternate scavenging circuitry and tapered cantilever beam," IEEE Trans. Ind. Electron., Mar. 2010, vol. 57, no. 3, pp. 820-830

[5] J. R. Liang and W. H. Liao, "Improved Design and Analysis of Self-Powered Synchronized Switch Interface Circuit for Piezoelectric Energy Harvesting Systems," J. Intell. Mater. Syst. Struct. , Mar. 2011, vol. 22, no. 5, pp. 503-512

[6] S. Roundy and P. K. Wright, "A Piezoelectric vibration based generator for wireless electronics", in Smart Mat. Struct. 2008, Vol. 13, pp. 1131-1142.

[7] G. K. Ottman, H. Hofmann and G. A. Lesieutre, "Optimized piezoelectric energy harvesting circuit using step-down converter in discontinuous conduction mode", IEEE Trans. Power Electron. 2003, Vol. 18, no. 2, pp. 696703.

[8] Na. Kong, T. Cochran, and D. Sam, "A Self-powered Power Management Circuit for Energy Harvested by a Piezoelectric Cantilever", in Applied Power Electronics Conference and Exposition (APEC), Feb. 2010, vol. 57, no. 3, pp. 2154-2160

[9] S. Corvo, "Sistema de Recolección de Energía Vibratoria con Parches Piezoeléctricos", PFC 2011, Universidad Politécnica de Madrid, ETSIA. 